\title{
Valor predictivo de los criterios de Beers y los criterios STOPP para detectar eventos adversos a medicamentos, consultas a emergencias y hospitalizaciones
}

Predictive value of the Beers criteria and the STOPP criteria for adverse drug events, emergency consultations and hospitalizations

\section{Objetivos}

Comparar el valor predictivo de los criterios de Beers 2003 y 2012 y los STOPP 2008 para detectar efectos adversos a medicamentos (EAM), consultas a emergencias (CE) y hospitalizaciones.

\section{Diseño, lugar y tiempo}

Estudio de Cohorte retrospectivo. Se relevaron registros de bases de datos secundarias de personas mayores de 65 años con al menos nueve meses de cobertura médica y farmacéutica, provenientes de 80 organizaciones de salud de los EE.UU., entre el año 2006 y 2009. El seguimiento fue hasta la ocurrencia de un evento o hasta el final del estudio.

\section{Prueba de estudio}

Se utilizaron herramientas para detectar la medicación potencialmente inadecuada (MPI). Se utilizaron los criterios de Beers 2003 y 2012 y los criterios de STOPP 2008. Se aplicaron a las historias clínicas de pacientes en las bases de datos relevadas. Se excluyeron las duplicaciones de medicamentos por no con-
Brown JD, y col. J Am Geriatr Soc. 2016;64:22-30. siderarse exclusivas de los pacientes mayores. Estos criterios tienen en cuenta las drogas que por sí solas en la población mayor son MPI, por interacción droga-enfermedad y condiciones clínicas en las cuales los medicamentos son potencialmente inadecuados.

\section{Resultados}

Se evaluaron 175.581 historias clínicas de pacientes mayores de 65 años que estuvieron al menos nueve meses de cobertura médica y farmacéutica, con un seguimiento medio de dos años (12 a 36 meses). Del total, $41,6 \%(n=72.493)$ presentó MPI de algunos de los tres sets de criterios; mientras que $19,7 \%$ presentó MPI en las tres herramientas. Comparativamente, los criterios Beers 2012 detectaron el $34,2 \%$, Beers 2003 el $32,2 \%$ y los criterios STOPP el $27,6 \%$ de MPI. En la tabla 1 se resume la distribución de los MPI según cada set de criterios, y en la tabla 2 , los resultados principales.Los criterios de Beers 2012 tuvieron mayor sensibilidad $(61,2$ a $71,2 \%$ ) y menor especificidad (42 a $70,1 \%)$, los STOPP tuvieron menor sensibilidad $(53,8 \%$ a $64,7 \%)$ pero mayor especificidad $(47,8 \%$ a $78,1 \%)$ para los eventos adversos.

Tabla 1. Distribución de los medicamentos potencialmente inapropiados detectados según criterios Beers 2003 y 2012 y los criterios STOPP.

\begin{tabular}{|c|c|c|c|}
\hline Set de Criterios & Beers 2012 & Beers 2003 & STOPP \\
\hline $\begin{array}{l}\text { Grupos de } \\
\text { medicamentos } \\
\text { (proporción) }\end{array}$ & $\begin{array}{l}\text {-IRSS y antipsicóticos asociados a hiponatremia por SIHAD }(16,2 \%) \\
\text {-Benzodiazepinas }(11,3 \%) \\
\text {-Relajantes musculares }(6,6 \%) \\
\text {-Hipnóticos no benzodiazepínicos (5,8\%) } \\
\text {-AINE }(5,4 \%)\end{array}$ & $\begin{array}{l}\text {-Anticolinérgicos y antihistamínicos }(19,4 \%) \\
\text {-IRSS }(10,5 \%) \\
\text {-Benzodiazepinas }(11,2 \%) \\
\text {-Relajantes musculares }(7,4 \%) \\
\text {-AINE }(5,1 \%)\end{array}$ & $\begin{array}{l}\text {-AINE }(16,2 \%) \\
\text {-Opioides }(4,8 \%) \\
\text {-Betabloqueantes }(4,7 \%) \\
\text {-Corticoides }(3,8 \%) \\
\text {-Antihistamínicos de 17a generación }(3,8 \%)\end{array}$ \\
\hline
\end{tabular}

IRSS: Inhibidor de recaptación selectiva de serotonina. SIHAD: Síndrome de secreción inadecuada de hormona antidiurética. AINE: antiinflamatorios no esteroideos.

Tabla 2. Resultados de ocurrencia de eventos según criterios Beers 2003, 2012 y STOPP.

\begin{tabular}{l|c|c|c}
\multicolumn{1}{c|}{ Tipo de evento } & $\begin{array}{c}\text { Beers 2012 } \\
\text { HR (IC 95\%) }\end{array}$ & $\begin{array}{c}\text { Beers 2003 } \\
\text { HR (IC 95\%) }\end{array}$ & $\begin{array}{c}\text { STOPP } \\
\text { HR (IC 95\%) }\end{array}$ \\
\hline Eventos adversos a medicamentos (EAM) & $2,51(2,33 \mathrm{a} 2,7)$ & $2,65(2,46 \mathrm{a} 2,85)$ & $2,89(2,68 \mathrm{a} 3,12)$ \\
\hline Consultas a emergencias (CE) & $2,21(2,16 \mathrm{a} 2,25)$ & $2,29(2,25 \mathrm{a} 2,34)$ & $2,66(2,60 \mathrm{a} 2,72)$ \\
\hline Hospitalizaciones & $2,25(2,20 \mathrm{a} 2,30)$ & $2,31(2,26 \mathrm{a} 2,37)$ & $2,80(2,74 \mathrm{a} 2,87)$ \\
\hline
\end{tabular}

HR: Hazard ratio. IC 95\%: intervalo de confianza del 95\%.

\section{Comentario}

El paciente mayor es especialmente vulnerable a la prescripción inadecuada $(\mathrm{PI})$ de medicamentos por muchas razones, entre las que se encuentran los cambios fisiológicos asociados a la edad, la polimedicación, la atención por múltiples especialistas y en dife-rentes niveles asistenciales, y algunas características de la interacción con el paciente en el momento de la prescripción ${ }^{1}$. Por estos motivos, suele excluirse a la población de edad avanzada de la mayoría de los estudios sobre eficacia y seguridad de los medicamentos $^{2,3}$. Sabemos que los pacientes ancianos tienen cuatro veces más riesgo de internación por EAM en comparación con pacientes más jóvenes ( $17 \%$ vs $4,1 \%$ ), y que el $88 \%$ de estas hospitalizaciones pueden ser prevenibles ${ }^{4}$. La PI y el inadecuado monitoreo son los motivos más frecuentes de los EAM prevenibles en estos pacientes ${ }^{5}$.

Por todo ello en las últimas dos décadas ha habido un creciente interés en la búsqueda de modalidades más objetivas para la detección de la prescripción potencialmente inadecuada (PPI). En los EE.UU. se desarrollaron los criterios de Beers, que se actualizaron en 2012 y 2015. Estos fueron adoptados por la Sociedad Americana de Geriatría y actualmente son ampliamente utilizados $^{6}$. En Europa, los más utilizados son los criterios STOPPSTART, desarrollados en Irlanda en 2008 y actualizados en $2014^{7}$. Trabajos realizados en Europa compararon los criterios STOPP 2008 con los Beers 2003 y observaron que los EAM potencial- mente evitables se debieron a medicamentos detectados por los criterios STOPP en el $67,7 \%$ de los casos, frente a solo el $28,5 \%$ de los casos señalados por los criterios de Beers. Las PPI detectadas por los criterios STOPP multiplicaban por 1,85 el riesgo de presentar un EAM, algo que no sucedía con los criterios de Beers $^{8}$. Utilizando los criterios STOPP, Cahir et al. encontraron que los pacientes con dos o más PPI tuvieron dos veces más riesgo de presentar EAM y de acudir al servicio de urgencias ${ }^{9}$.

Este es el primer trabajo que compara estos criterios en los EE.UU. para detectar asociación de riesgo entre MPI y estos eventos (EAM, CE y hospitalización por todas las causas), encontrándose un aumento del riesgo entre dos a tres veces entre quienes presentan estos criterios y quienes no los presentan. Es un estudio de cohorte retrospectivo bien diseñado donde se cuidó la relación temporal entre la detección de MPI y los eventos. Los distintos criterios utilizados detectaron MPI entre el 27 y el $34 \%$. Los STOPP fueron más sensibles para detectar AINES y opiáceos, lo que podría explicar que sean más específicos para detectar los eventos evaluados. Los Beers fueron más sensibles para detectar psicofármacos (inhibidores de recaptación selectiva de serotonina, antipsicóticos y benzodiacepinas). La combinación de ambos criterios aumentó la sensibilidad y especificidad para la detección de eventos.

Se debe tener en cuenta que las CE y las internaciones fueron 
por todas las causas y no solamente por EAM. En los trabajos donde las internaciones son por EAM en adultos mayores, como el pu-blicados por Budnitz y col., se vio que los medicamentos más implicados eran los antagonistas de la vitamina $\mathrm{k}(33,3 \%)$, la insulina $(13,3 \%)$, los antiplaquetarios $(13,3 \%)$, los hipoglucemiantes orales (10,7\%), los opiáceos (5\%) y los antibióticos (4\%); siendo responsables del $76 \%$ de las internaciones. La detección de MPI por los criterios Beers 2003 fue responsable del 1,2\% de las internaciones, de las cuales el $50 \%$ involucró a la digoxina ${ }^{10}$. Las últimas versiones de Beers y STOPP podrían aumentar su sensibilidad.

Debemos considerar que estas herramientas no tienen en cuenta las interacciones medicamentosas y el monitoreo de medicamentos de alto riesgo, situaciones frecuentemente implicadas en las internaciones por EAM.

\section{Conclusiones del comentador}

Como podemos ver estas herramientas son útiles ya que son objetivas y fáciles de aplicar, pero no reemplazan el juicio clínico. Sirven como una guía para la prescripción racional de medicamentos donde es clave también tener en cuenta la expectativa de vida, el tiempo hasta el beneficio del fármaco, las metas de cuidado y las preferencias del paciente.

Cristian Gallo [ Sección Medicina domiciliaria, Servicio Clínica Médica del Hospital Italiano de Buenos Aires cristian.gallo@ hospitalitaliano.org.ar ] Bruno Boietti [ Área de Investigación en medicina interna, Servicio Clínica Médica del Hospital Italiano de Buenos Aires ]

Gallo C, y Boietti B. Valor predictivo de los criterios de Beers y los criterios STOPP para detectar eventos adversos a medicamentos, consultas a emergencias y hospitalizaciones. Evid Act Pract Ambul. 2017;20(2):49-50. Comentado de: Brown JD. Predictive Validity of the Beers and Screening Tool of Older Persons' Potentially Inappropriate Prescriptions (STOPP) Criteria to Detect Adverse Drug Events, Hospitalizations, and Emergency Department Visits in the United States. J Am Geriatr Soc. 2016;64:22-30. PMID: 26782849.

\section{Referencias}

1. Page RL, y col. Inappropriate prescribing in the hospitalized elderly patient: defining the problem, evaluation tools, and possible solutions. Clin Interv Aging. 2010 Apr 7;5:75-87. 2. Wynne HA, y col. Drug treatment in an ageing population: practical implications. Maturitas. 2010 Jul:66(3):246-50.

3. Borchelt M. Important aspects of geriatric pharmacotherapy. Bundesgesundheitsblatt Gesundheitsforschung Gesundheitsschutz 2005:48(5):593-8.

4. Beijer HJ, y col. Hospitalisations caused by adverse drug reactions (ADR): a meta-analysis of observational studies. Pharm World Sci 2002;24(2):46-54

5. Gurwitz JH, y col. The incidence of adverse drug events in two large academic long-term care facilities. Am J Med 2005; 118(3):251-8.

6. Fick DM, y col. 2012 American Geriatrics Society Beers Criteria: new year, new criteria, new perspective. J Am Geriatr Soc. 2012 Apr;60(4):614-5.

7. Delgado Silveira E, y col. Improving drug prescribing in the elderly: a new edition of STOPP/START criteria. Rev Esp Geriatr Gerontol. 2015 Mar-Apr;50(2):89-96.

8. Mimica Matanovic S, y col. Potentially inappropriate medications in the elderly: a comprehensive protocol. Eur J Clin Pharmacol. 2012 Aug;68(8):1123-38.

9. Cahir C, y col. Potentially inappropriate prescribing and adverse health outcomes in community dwelling older patients. Br J Clin Pharmacol. 2014 Jan;77(1):201-10.

10. Budnitz DS, y col. Emergency hospitalizations for adverse drug events in older Americans. N Engl J Med. 2011 Nov 24;365(21):2002-12

\section{¡Vos también podes formar parte de esta transformación!}

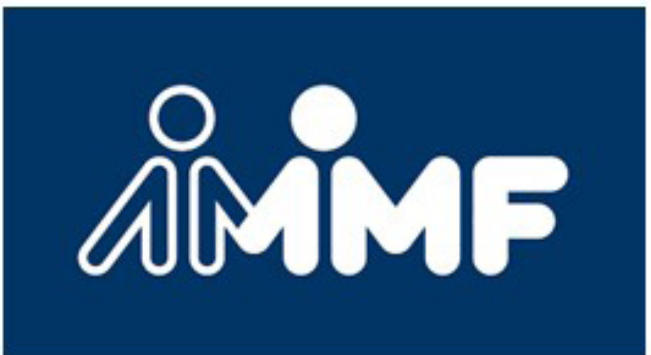

Asociación Metropolitana de Medicina Familiar.

Si queres asociarte o que te brindemos mayor información: secretaria.ammf@gmail.com Seguinos en Facebook: www.facebook.com/AsociacionMetropolitanadeMedicinaFamiliar 